\title{
Influence of Personality on Shape-Based Design Activities
}

\author{
Stefano Filippi $(\mathbb{D}$ and Daniela Barattin \\ DPIA Department, University of Udine, 33100, Udine, Italy \\ Correspondence should be addressed to Stefano Filippi; filippi@uniud.it \\ Received 31 May 2018; Revised 11 December 2018; Accepted 31 March 2019; Published 2 May 2019 \\ Academic Editor: Francesco Bellotti
}

Copyright ( 2019 Stefano Filippi and Daniela Barattin. This is an open access article distributed under the Creative Commons Attribution License, which permits unrestricted use, distribution, and reproduction in any medium, provided the original work is properly cited.

\begin{abstract}
As the literature demonstrates, designers' personality influences design activities like different ways to represent environments and/or products, technological advances, etc.. Nevertheless, an exhaustive analysis on the influence of personality on design activities involving different representations is missing. This research explores this gap by studying this influence on specific design activities, the shape-based ones (i.e., analysis of specific shapes and highlighting of functions suggested by them). People showing different personalities undergo tests where they carry out design activities exploiting several representations. The results confirm the influence of personality on shape-based design activities and allow highlighting different keys to interpret and exploit these results. Thanks to the results of this study, researchers can increase their knowledge about subjective aspects of design as well as about how these aspects coexist with classic and emerging representations. As well, designers can try to maximize the effectiveness of their efforts by selecting the best combinations of personality, representations, and characteristics of the expected design results time by time.
\end{abstract}

\section{Introduction}

The literature already demonstrates the influence of human personality on design. Sung and Choi [1] show that extraversion and openness to experience/culture positively influence creativity because people with these traits are more flexible and able to analyse ideas from different perspectives. Filippi and Barattin [2] confirm this and report also that conscientiousness and agreeableness positively influence the number of design solutions and their pertinence against the specific context because people showing these traits work profitably together to reach their goals. Steel et al. [3] claim that conscientiousness tends to increase innovation. This happens because innovation requires hard work, mainly to bring inventions to successful adoptions; conscientious people, strongly oriented to the goal, seem the most suitable in this case. Rothmann and Coetzer [4] highlight that people who tend towards neuroticism find less design solutions, with lower creativity, than people emotionally stable do. This happens because neurotic people are prone to having irrational ideas, are less able to control their impulses, and cope poorly with stress. Kohn and Smith [5] show that during a brainstorming process, agreeable people generate fewer ideas, with lower variety, because of the influence of the other participants.

The literature shows also that different ways to represent environments, products, interactions between environments and products, users, and interactions between products and users [6] influence design activities. According to Starkey et al. [7], virtual and real representations of products can affect creativity differently. Considering product dissection as a help to generate results for a design problem, tests with real users prove that dissection of virtual products rather than real ones increases creativity by reducing the effects of fixation. To Maher et al. [8], real and tangible interfaces allowing direct interactions encourage users' creativity and increase the number of design solutions, all of this in comparison to indirect interactions where pointing interfaces are used. Youmans [9] demonstrates that using physical prototypes instead of virtual ones improves novelty of design solutions. Kohler et al. [10] highlight that using simulated users (avatars) instead of real ones during interaction with virtual products in virtual environments can increase product innovation because all of this allows designers to work remotely and asynchronously. Filippi and Barattin [6] demonstrate the 
influence of three different representations on specific design activities with respect to the quantity, variety, novelty, and usefulness metrics. For example, they find that virtual products and environments increase variety and novelty of design solutions, while real products and environments increase quantity and usefulness.

Nevertheless, an exhaustive analysis of the influence of personality on design activities involving different representations is missing. In trying to fill this gap, the research described in this paper explores this influence on specific design activities, the shape-based ones (i.e., analysis of specific shapes and highlighting of functions suggested by them) [11]. The selection of this type of activities lies on one hand on the fact that they are affordable in terms of costs and resources when performed in user tests; on the other hand, they can be performed even by people showing low/medium design skill and knowledge. By considering the results of this study and applying them to different design activities in different contexts, researchers can increase their knowledge about subjective aspects of design as well as about how these aspects coexist with classic and emerging representations. As well, designers can try to maximize the effectiveness of their efforts by selecting the best combinations of personality, representations, and characteristics of the expected design results time by time.

The results obtained by Filippi and Barattin in $[2,6]$ are the starting points of this research. Similar tests on the same design activities (the shape-based ones), using the same representations and involving different users' personalities are conducted. The well-known big five personality traits taxonomy [4] is used to describe testers' personalities. Testers highlight functions and behaviours that two shapes suggest to them. After that, quantitative and qualitative analyses of these results with respect to quantity, variety, novelty, and usefulness metrics take place. At the end, some reasoning on the results proposes different ways to interpret and exploit them in the field.

The paper goes on with the background section summarizing the definition of personality, the classification of representations, and the description of the shape-based design activities. The activities section describes the development and execution of the tests as well as the data analysis, including the adoption of the specific metrics, and the validation, interpretation, and exploitation of the results. The conclusions, together with some perspectives on future work, close the paper.

\section{Background}

This section summarizes the needs of this research; their wider description can be found in $[2,6]$. What appears here is just to get the reading of the paper easier and feasible. The section starts by reporting the definition of the personality traits, followed by the classification of representations and the description of the shape-based design activities.

2.1. The Big Five Personality Traits. Human personality can be defined as the set of characteristics of a person that account for consistent behavioural patterns over situations and time [12]. Starting from several researches in the literature [1317], the big five personality traits, this is the name of their recognized taxonomy, are defined in $[2,4]$ and summarized as follows. Extraversion or surgency (Personal Trait 1 PT1): extraverts are energetic and optimistic; introverts are reserved rather than unfriendly, independent rather than followers. Agreeableness (PT2): an agreeable person is fundamentally altruistic, sympathetic to others and eager to help; the disagreeable/antagonistic person is egocentric, sceptical of others' intentions and competitive. Conscientiousness (PT3): a conscientious person is purposeful, strong-willed, and determined; low scorers on conscientiousness may not necessarily lack moral principles, but they are less exacting in applying them. Neuroticism (PT4): neurotic people suffer from fear, sadness, embarrassment, anger, etc.; low neuroticism is indicative of emotional stability. Openness to experience/culture (PT5): open individuals are curious about both inner and outer worlds and their lives are experientially richer; people scoring low tend to be conventional in behaviour and conservative in outlook.

2.2. Classification of Representations. Several researches classify different ways to represent products, environments, users, etc. Filippi and Barattin [6] make an in-depth analysis on these researches and offer a univocal classification of them based on five elements. These elements are orthogonal, they can cover both classic and more recent product development processes, and they are clearly stated thanks to discrete values assignable to them. These elements are the environment, the product, the interaction between environment and product, and the user and the interaction between product and user. Figure 1 shows the classification of representations (please ignore the grey paths for the moment). This classification consists of combinations of the values the five elements can assume. The resulting representations are Virtual Reality (VR), Augmented Virtuality (AV), Augmented Reality (AR), Pure Reality (PR), and Mixed Reality (MR).

2.3. Shape-Based Design Activities. Shape-based design activities develop products by analysing specific shapes and defining product functions and behaviours consequently [6, 11]. One of the main goals is to arouse specific emotions in the people who will interact with those products. This type of design activities appears in both the academic and industrial fields. For example, in the academic field, Mikkonen and Hsieh study these activities to develop a design approach for deformable interfaces of mobile devices [18]. They perform tests involving designers and final users. At the beginning, designers touch blindly some mock-ups of deformable interfaces and express their sensations. Then, the mock-ups are evaluated using sight, without and with considering a specific context where designers are called to empathize with those mock-ups. Designers also ask final users to perform the same activities to get more data about the sensorial experience. At the end of the evaluation, designers express which functions and behaviours the mock-ups inspired and those eventually disliked. These outcomes are considered to improve the 


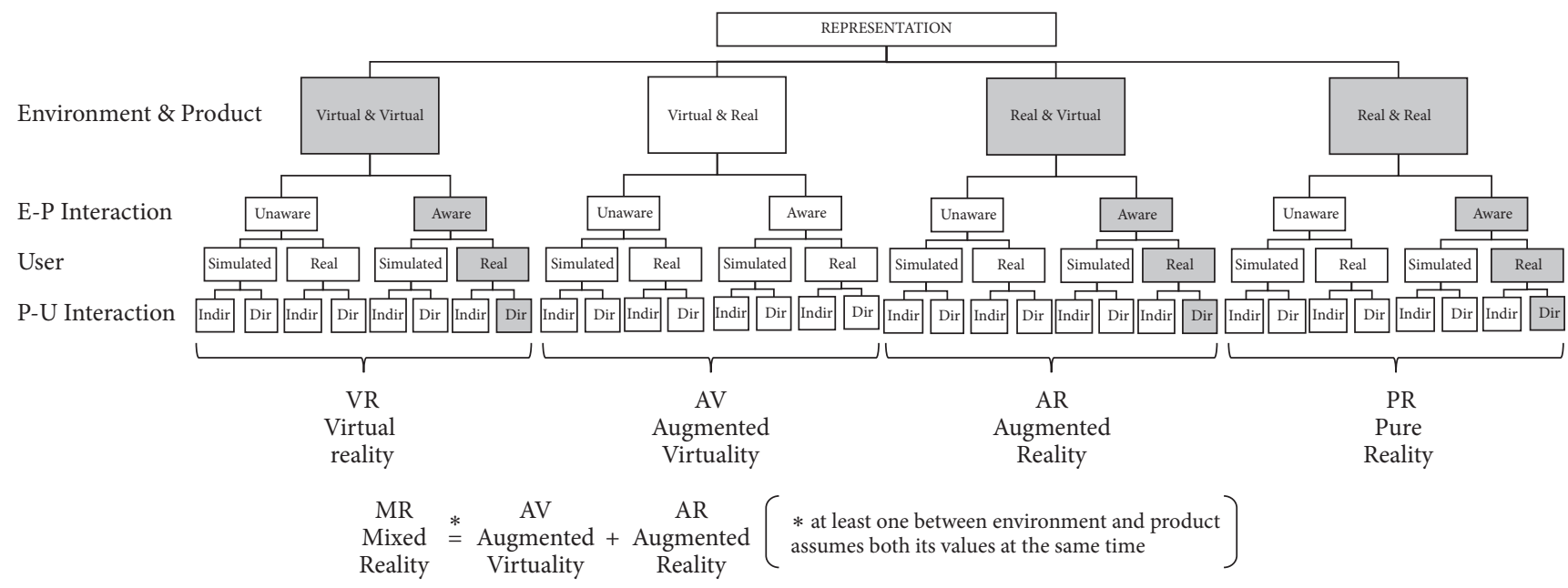

FIGURE 1: Classification of representations.

mock-ups for the specific case study. Then, new prototypes are generated and compared with the previous ones to highlight the differences and suggest possible improvements. All of this allows Mikkonen and Hsieh developing and tuning up their design approach that, once available, will be transferred to the industrial field and used by a big company. In the industrial field, Alessi, an Italian company producing iconic objects, mainly home appliances, bases its activities on the analysis of shapes generated by fashion designers [19]. Figure 2 shows three well-known Alessi products: the spaghetti measure VOILE, the citrus-squeezer Juicy Salif, and the orange peeler Apostrophe. They are clear examples of the shape-to-function approach to design. The first shape could resemble some jewellery, the second a rocket, and the third a drop or a sack; there is nothing in common with their final destination of usage. Functions have been set by analysing those shapes as they were, keeping just emotions into consideration and exploring as many application contexts as possible.

This research considers only the first part of the shapebased design activities, the shape analysis. Shape analysis usually takes place thanks to tests where interaction involves touch and sight. This interaction highlights emotions and behaviours of people as well as possible functions and behaviours of products shaped that way. After that, the most promising functions and product behaviours are implemented in order to arouse the same emotions and allow the same people's behaviours.

\section{Activities}

The research activities start from the work published in [6] and develop it further by involving personality. At the beginning, the definition of the possible values the personality traits can assume here as well as the selection of the representations to consider and of the metrics for the data analysis takes place. Then, the activities carry on by developing three user tests; each test considers the same two shapes and performs the same shape-based design activities but involves a different representation. Three experts in product development processes collect and analyse the data and validate their reliability using statistical methods. Finally, the last activity highlights two interpretation keys of the results and some possible exploitations of them in the field.

\subsection{Definition of the Possible Values of the Personality Traits.} This research considers only two possible values for each personality trait. These values are as follows: extraverted and introverted for PT1, agreeable and disagreeable for PT2, conscientious and unconscientious for PT3, neurotic and unneurotic for PT4, and open to experience and closed to experience for PT5. This coarse choice comes from the desire to develop, implement, and verify all the steps required to get some first results as quickly as possible. A finer quantification of the personality traits is already in the to-do list for future work.

3.2. Selection of the Representations to Consider. As in [6], VR, $\mathrm{AR}$, and $\mathrm{PR}$ are the representations selected to perform the shape-based activities in the tests, considering for all of them the interaction between environment and product aware, the users real, and the interaction between product and user direct. These representations are chosen again because, from one side, they appear as enough to get meaningful results and their potentialities and coverage are witnessed by many researches in different application fields [20-22]; on the other side, $\mathrm{AV}$ and $\mathrm{MR}$, the other possible representations, require expensive tools to represent the environment (projectors, big screens, rooms, etc.) and specific resources to develop complex procedures to make the environment and/or the product real and virtual at the same time.

3.3. Selection of the Metrics. Once facing the shapes represented as VR, AR, or PR, the testers will report all those functions that the shapes suggest to them, imagining the interaction with products shaped those ways. For each function, they will also describe their own behaviours and those of 

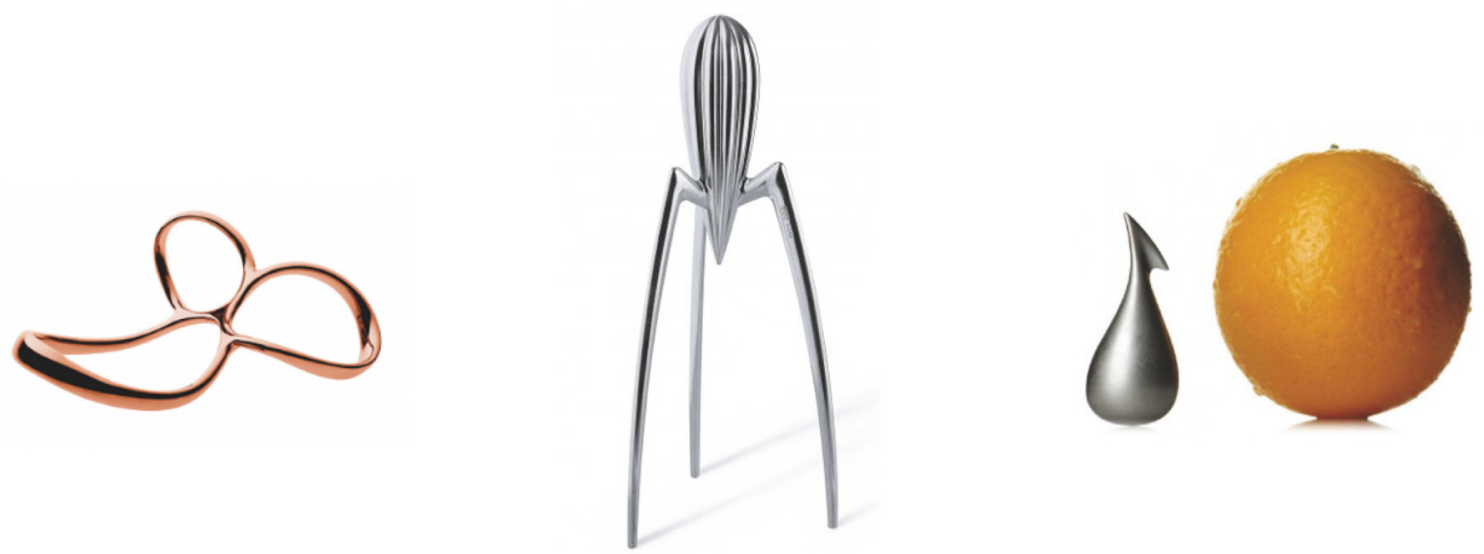

FIgURE 2: Three well-known Alessi products.

TABLE 1: Characteristics of the two shapes used for the tests.

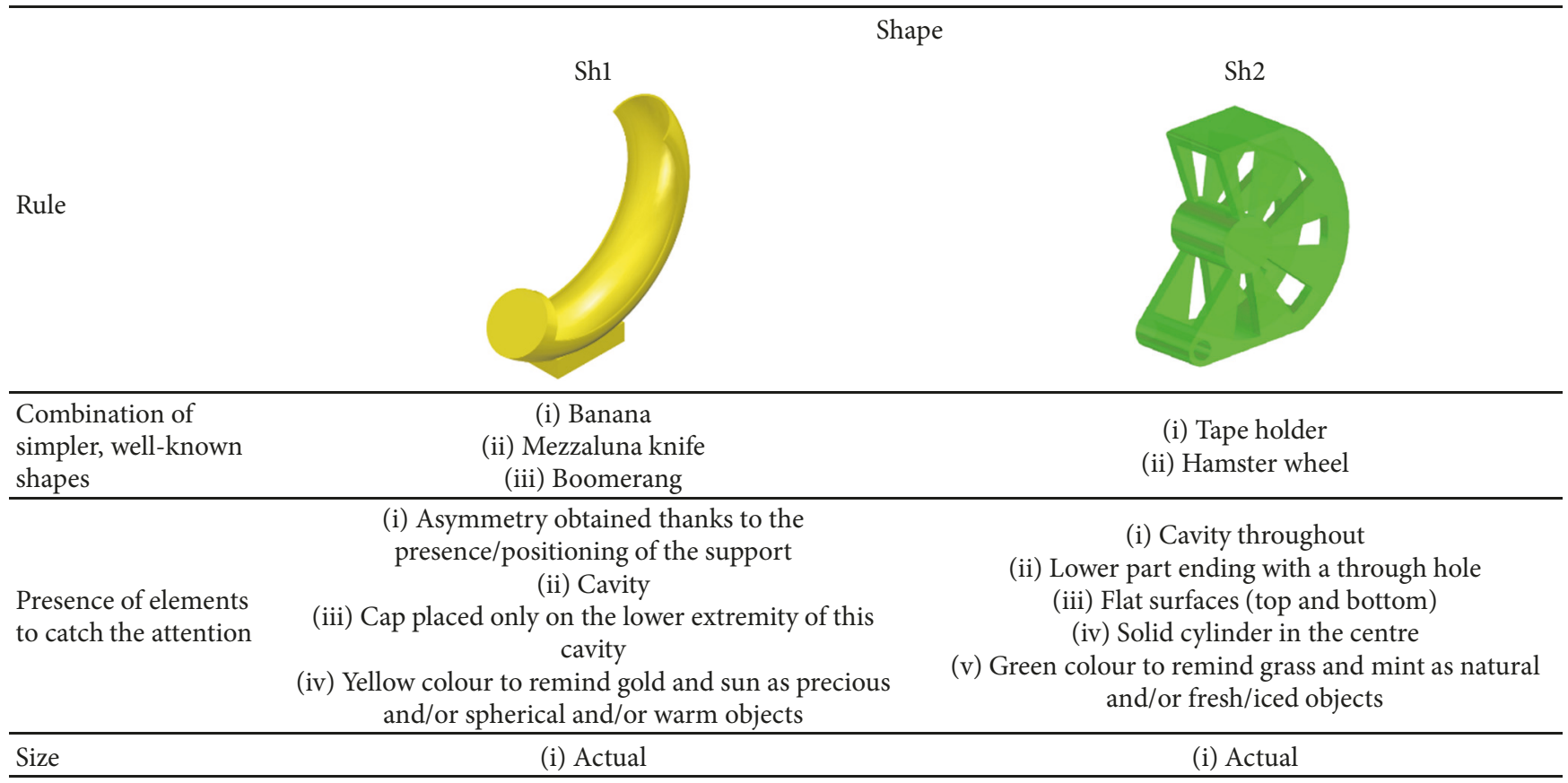

the product, both aimed at implementing that function. These data appear as function/behaviours (F/B) pairs. Filippi and Barattin [6] suggest the four metrics to analyse these data and evaluate the influence of personality and representations on shape-based design activities. These metrics are the quantity, variety, novelty, and usefulness. Creativity metrics is usually considered as well but it does not appear explicitly in this research because, as stated by Sarkar and Chakrabarti [23], creativity is just the product of novelty and usefulness, already considered in detail here.

3.4. Test Setup and Execution. What follows reports in detail the new activities and material with respect to what is described in [6]. All the rest is just summarized again to get the reading of the paper easier and feasible.
3.4.1. Preparation of the Material to Conduct the Tests. The preparation of the material regards the shapes and the documents used in the tests. Each tester will interact with the same two shapes, labelled as Sh1 and Sh2 in the following. This research uses more than one shape in order to minimize the bias due to specific shape characteristics. Three rules, as described in [11], lead the definition of these shapes. The first rule requires the shape consisting of a combination of simpler, well-known shapes; the second rule claims that the shape must involve elements to catch the attention and suggest as many F/B pairs as possible; at the same time, the number of these elements must be limited to avoid bias; finally, the third rule imposes the shape size as the actual one for the same reason. Table 1 summarizes the characteristics of the two shapes in answering to the three rules. 


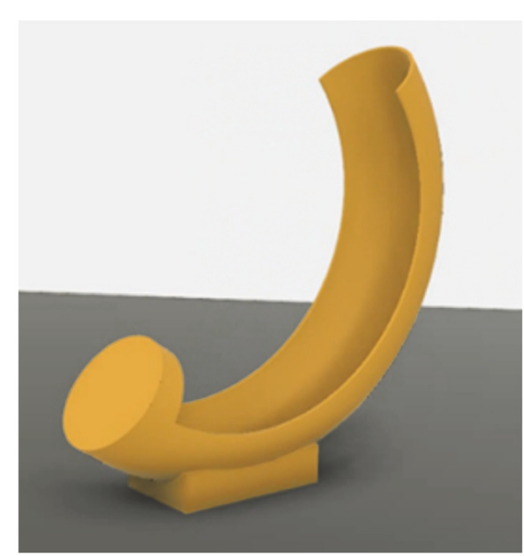

(a)

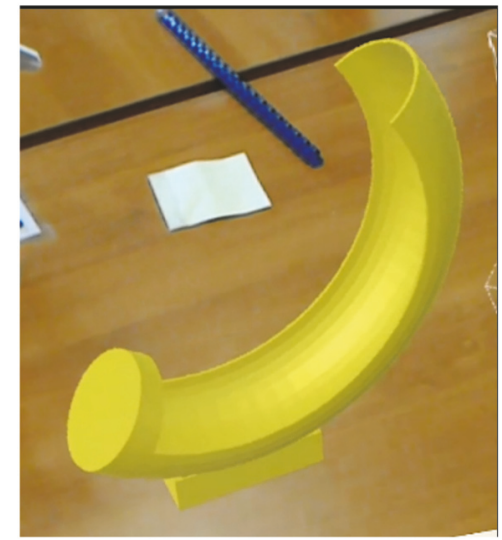

(b)

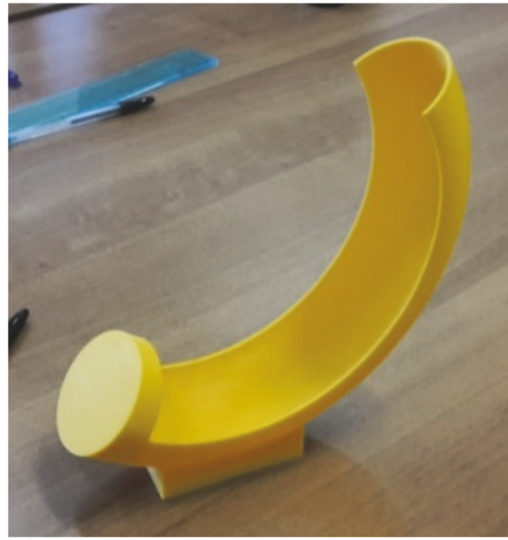

(c)

FIgURE 3: Representations of Shl and environment as used in VR (a), AR (b), and PR (c) tests.

The Fusion 360 CAD software package by AutoDesk is used to generate the models of shapes and desk for the VR test; testers rotate these models with the Microsoft 3D Builder software package. Then, the CAD models of the shapes are converted into holograms through the software packages Unity by Unity Technologies, Blender by Blender Foundation, and Microsoft Visual Studio, to be projected on a real desk by the Microsoft HoloLens device [24] in the AR test. Finally, the physical models of the shapes are built using the 3D printer Ultimaker 2 by Ultimaker [25] for the PR test. Once built, the physical models are placed on the same real desk used in the AR test. Figure 3 shows the representations of Shl and environment as used in VR (a), AR (b), and PR (c) tests.

The documents for the testers (test user guides, hereafter) describe each design activity to perform using nontechnical, tester-oriented language. These guides also report the design problem "express functions and behaviours that specific shapes suggest to you" and an example involving a cup for coffee with the handle positioned inside rather than outside. The test user guides contain empty tables to write down the F/B pairs and free spaces for additional comments about them.

3.4.2. Execution of the Tests and Collection of the Data. Once the material is ready and available, the selection of the testers takes place. Students of Mechanical Engineering courses are involved because of their similar skill and knowledge about design processes, methods, and tools, all of this to avoid bias. Clearly, skill and knowledge influence design activities but these factors are not considered here; they will be kept into consideration in future work. Fifty-six students receive a questionnaire by e-mail, aiming at knowing their personality. This questionnaire is the Big Five Inventory (BFI) [26]. The BFI consists of forty-four items that testers mark by assigning values between 1 (strongly disagree) and 5 (strongly agree). The analysis of the answers computes a 0 to 100 score for each personality trait. Finally, scores are split into two intervals: values in the interval $(50 . .100]$ drive to the trait itself and those in the interval [0..50] to its opposite. Fiftythree students answer and all of them are recruited for the tests. The questionnaires allow distributing the students in the three tests to have similar numbers of testers for each personality trait in order to guarantee the comparability of the results. For example, the VR test involves twelve extraverted and nine introverted people; agreeable testers are ten while disagreeable ones are eleven. The distribution leads to this result: twenty-one testers perform the VR test, seventeen the AR test, and fifteen the PR test. Tests take place in a university lab, one tester at a time. At the beginning, the tester receives the test user guide. After that, the first shape is unveiled; the tester has ten minutes to analyse it seeing and moving around (virtually or really, due to the type of test) without touching it and to write down the F/B pairs that come to his/her mind. Then, the experts unveil the second shape and the tester has ten minutes again to analyse it and write down the F/B pairs. At the end, the tester returns the test user guide to the three experts.

3.5. Test Results. This section reports the elaboration of the data, from the adoption of the metrics up to the validation, interpretation, and possible exploitations of the results. It is worth to say that the data analysis considers personality traits as independent from each other. This allows performing the analysis without investigating on relationships among them, which would require a much deeper reasoning from the psychological point of view. Nevertheless, the study of this aspect will occur in future work.

3.5.1. Adoption of the Metrics. The adoption of the metrics occurs in two steps. First, the quantity values to each tester and those of variety, novelty, and usefulness to each F/B pair are assigned. After that, the computation of the mean value for each metrics for each value of each personality trait in each test takes place, considering the $\mathrm{F} / \mathrm{B}$ pairs coming from both the shapes. For example, the mean value of the variety metrics is computed for all the extraverted testers in the VR test 
TABLE 2: Results of the tests.

\begin{tabular}{|c|c|c|c|c|c|c|c|c|c|c|c|c|c|}
\hline \multirow{3}{*}{\multicolumn{2}{|c|}{ Personality traits }} & \multicolumn{12}{|c|}{ Metrics/Representations } \\
\hline & & \multicolumn{3}{|c|}{ Quantity $[0,+\infty]$} & \multicolumn{3}{|c|}{ Variety $[1,10]$} & \multicolumn{3}{|c|}{ Novelty $[0,1]$} & \multicolumn{3}{|c|}{ Usefulness $[0,1]$} \\
\hline & & VR & $\mathrm{AR}$ & PR & VR & $\mathrm{AR}$ & PR & VR & $\mathrm{AR}$ & PR & VR & AR & PR \\
\hline \multirow{2}{*}{ PT1 } & Extravert & 3.5 & 4.1 & 5.1 & 9.82 & 9.51 & 9.75 & 0.36 & 0.41 & 0.35 & 0.045 & 0.061 & 0.058 \\
\hline & Introvert & 2.4 & 3.8 & 4.2 & 9.65 & 9.44 & 9.58 & 0.33 & 0.32 & 0.36 & 0.048 & 0.061 & 0.078 \\
\hline \multirow{2}{*}{ PT2 } & Agreeable & 3.5 & 4.5 & 3.2 & 9.61 & 9.72 & 10.0 & 0.41 & 0.36 & 0.31 & 0.042 & 0.058 & 0.072 \\
\hline & Disagreeable & 2.8 & 3.4 & 5.3 & 10.0 & 9.85 & 9.71 & 0.32 & 0.33 & 0.35 & 0.055 & 0.054 & 0.054 \\
\hline \multirow{2}{*}{ PT3 } & Conscientious & 3.5 & 4.3 & 5.4 & 9.91 & 9.65 & 9.53 & 0.35 & 0.37 & 0.33 & 0.024 & 0.049 & 0.098 \\
\hline & Unconscientious & 1.4 & 4.5 & 3.6 & 8.81 & 9.65 & 8.22 & 0.25 & 0.33 & 0.36 & 0.103 & 0.057 & 0.074 \\
\hline \multirow{2}{*}{ PT4 } & Neurotic & 3.0 & 4.5 & 4.8 & 9.79 & 9.69 & 9.48 & 0.41 & 0.38 & 0.31 & 0.032 & 0.065 & 0.072 \\
\hline & Unneurotic & 1.8 & 3.5 & 4.4 & 9.15 & 9.77 & 9.65 & 0.32 & 0.38 & 0.36 & 0.073 & 0.039 & 0.048 \\
\hline \multirow{2}{*}{ PT5 } & Open to experience & 3.8 & 3.2 & 4.8 & 9.75 & 9.62 & 9.61 & 0.39 & 0.35 & 0.34 & 0.029 & 0.057 & 0.039 \\
\hline & Closed to experience & 2.4 & 4.9 & 4.2 & 9.81 & 9.66 & 9.43 & 0.33 & 0.35 & 0.39 & 0.058 & 0.043 & 0.048 \\
\hline
\end{tabular}

considering the F/B pairs from Sh1 and Sh2. The computation of the mean values is the same for each metrics; the only difference is in how the number of testers is considered. Specifically, the adoption of each metrics comes as follows.

(i) Quantity: number of F/B pairs expressed by each tester. The computation of the mean value considers the number of testers sharing the same value for every personality trait for each representation.

(ii) Variety: value assigned to each $\mathrm{F} / \mathrm{B}$ pair; it ranges from 1 to 10 . If all the testers sharing the same value for every personality trait for each representation express that $\mathrm{F} / \mathrm{B}$ pair, the variety will be 1 ; if only one tester expresses it, the variety will be 10 . A simple formula allows assigning all the other values in between. The computation of the mean value ignores the number of testers because this has been already considered in every assignment of the 1 to 10 values.

(iii) Novelty: value assigned to each $\mathrm{F} / \mathrm{B}$ pair; it ranges from 0 to 1 . Novelty is equal to 0 if that F/B pair is already present in one or more existing products as it is. On the contrary, the value is 1 if the $\mathrm{F} / \mathrm{B}$ pair is not present in any existing product. All the other values in between are assigned due to considerations about the presence and implementation of the F/B pair. The computation of the mean value ignores the number of testers because novelty depends from external factors (existing products).

(iv) Usefulness: value assigned to each $\mathrm{F} / \mathrm{B}$ pair; it is the product of the level of importance, the rate of popularity of usage, and the rate of duration of benefit. The level of importance, representing the impact of F/B pair on testers' life, varies from 0 - to 1 . The rate of popularity of usage is the ratio between the number of testers sharing thevsame value for every personality trait for each representation who expressed a specific $\mathrm{F} / \mathrm{B}$ pair and the total number of them. Thus, its value is in the range $[0,1]$. The rate of duration of benefit is the percentage of time the tester spends with that $\mathrm{F} / \mathrm{B}$ pair. Once normalized, its value is in the range $[0,1]$ as well. The computation of the usefulness mean value ignores the number of testers because this has been already considered during the assignment of the rate of popularity of usage.

Table 2 contains the results of the tests. The ranges used for representing the metrics values appear in the table to recall their heterogeneity (due to the fact that the original ways to compute them are used) and to highlight once again the impossibility of comparing the metrics to each other.

3.5.2. Validation of the Test Results. The results of the tests undergo statistical analyses using two t-tests (test of Student) [27]. The first t-test verifies possible influences of shape characteristics on results. This is required to claim that the content of Table 2 is free from that kind of bias. The second analysis verifies the influences of personality traits on the results. The low number of tests performed suggests waiting for further data before applying in-depth statistical analyses and related interpretation of results. For this reason, the goal of the two t-test adoptions described in the following is just to start demonstrating the robustness of the test results.

The first analysis considers the null hypothesis "the means of the results achieved considering each shape for the same personality trait, metrics and representation are equal"; in other words, these means are just affected by randomness and not by specific shape characteristics. The alternative hypothesis consists in asserting that the means are different. The suitable type of t-test is the two-sided paired one because the groups of testers who generate the results considering the two shapes are the same. To verify if the null hypothesis is rejected or not, the calculation of the $P$-value takes place. The $P$-value is the smallest level of significance that would lead to the rejection of the null hypothesis with the given data. Once computed, the $P$-value is compared to a significance level $\alpha$, usually equal to 0.05 or lower [27]. The significance level here is set as 0.1 because of the low number of testers. Table 3 reports the $P$-values computed for this first analysis. All the values are bigger than $\alpha$; therefore, the null hypothesis cannot be rejected and the characteristics of the shapes used in the tests do not have influence on the test results. 
TABLE 3: P-values of the first t-test analysis focused on possible influences of shapes on design results.

\begin{tabular}{|c|c|c|c|c|c|c|c|c|c|c|c|c|c|}
\hline \multirow{3}{*}{\multicolumn{2}{|c|}{ Personality traits }} & \multicolumn{12}{|c|}{ Metrics/Representations } \\
\hline & & \multicolumn{3}{|c|}{ Quantity } & \multicolumn{3}{|c|}{ Variety } & \multicolumn{3}{|c|}{ Novelty } & \multicolumn{3}{|c|}{ Usefulness } \\
\hline & & VR & $\mathrm{AR}$ & $\mathrm{PR}$ & VR & $\mathrm{AR}$ & $\mathrm{PR}$ & VR & $\mathrm{AR}$ & PR & VR & $\mathrm{AR}$ & $\mathrm{PR}$ \\
\hline \multirow{2}{*}{ PT1 } & Extravert & 0.76 & 0.82 & 0.69 & 0.41 & 0.37 & 0.39 & 0.85 & 0.87 & 0.76 & 0.36 & 0.32 & 0.32 \\
\hline & Introvert & 0.76 & 0.53 & 0.82 & 0.91 & 0.73 & 0.81 & 0.36 & 0.52 & 0.59 & 0.82 & 0.34 & 0.60 \\
\hline \multirow{2}{*}{ PT2 } & Agreeable & 0.54 & 0.81 & 0.67 & 0.79 & 0.64 & 0.68 & 0.78 & 0.24 & 0.41 & 0.43 & 0.56 & 0.50 \\
\hline & Disagreeable & 0.98 & 0.76 & 0.55 & 0.88 & 0.90 & 0.69 & 0.61 & 0.54 & 0.55 & 0.88 & 0.73 & 0.88 \\
\hline \multirow{2}{*}{ PT3 } & Conscientious & 0.55 & 0.81 & 0.75 & 0.51 & 0.58 & 0.53 & 0.82 & 0.80 & 0.83 & 0.58 & 0.56 & 0.91 \\
\hline & Unconscientious & 0.81 & 0.78 & 0.79 & 0.73 & 0.42 & 0.37 & 0.81 & 0.58 & 0.80 & 0.81 & 0.42 & 0.66 \\
\hline \multirow{2}{*}{ PT4 } & Neurotic & 0.42 & 0.83 & 0.47 & 0.82 & 0.62 & 0.60 & 0.81 & 0.89 & 0.33 & 0.57 & 0.60 & 0.83 \\
\hline & Unneurotic & 0.31 & 0.43 & 0.40 & 0.82 & 0.81 & 0.43 & 0.59 & 0.82 & 0.39 & 0.72 & 0.60 & 0.66 \\
\hline \multirow{2}{*}{ PT5 } & Open to experience & 0.80 & 0.75 & 0.80 & 0.72 & 0.58 & 0.67 & 0.81 & 0.80 & 0.43 & 0.47 & 0.54 & 0.70 \\
\hline & Closed to experience & 0.75 & 0.58 & 0.91 & 0.73 & 0.58 & 0.62 & 0.29 & 0.32 & 0.33 & 0.48 & 0.45 & 0.43 \\
\hline
\end{tabular}

TABLE 4: P-values of the second t-test analysis focused on possible influences of personality traits on design results.

\begin{tabular}{|c|c|c|c|c|c|c|c|c|c|c|c|c|}
\hline \multirow{3}{*}{ Personality traits } & \multicolumn{12}{|c|}{ Metrics/Representations } \\
\hline & \multicolumn{3}{|c|}{ Quantity } & \multicolumn{3}{|c|}{ Variety } & \multicolumn{3}{|c|}{ Novelty } & \multicolumn{3}{|c|}{ Usefulness } \\
\hline & VR & $\mathrm{AR}$ & PR & VR & $\mathrm{AR}$ & PR & VR & $\mathrm{AR}$ & PR & VR & $\mathrm{AR}$ & PR \\
\hline PT1 & 0.08 & 0.03 & 0.05 & 0.02 & 0.06 & 0.08 & 0.09 & 0.05 & 0.05 & 0.18 & 0.10 & 0.14 \\
\hline PT2 & 0.06 & 0.08 & 0.09 & 0.08 & 0.07 & 0.06 & 0.07 & 0.08 & 0.07 & 0.07 & 0.12 & 0.09 \\
\hline PT3 & 0.07 & 0.08 & 0.05 & 0.10 & 0.08 & 0.08 & 0.07 & 0.08 & 0.08 & 0.08 & 0.08 & 0.06 \\
\hline PT4 & 0.08 & 0.09 & 0.10 & 0.09 & 0.06 & 0.09 & 0.09 & 0.07 & 0.09 & 0.05 & 0.09 & 0.07 \\
\hline PT5 & 0.10 & 0.07 & 0.12 & 0.06 & 0.05 & 0.11 & 0.06 & 0.10 & 0.12 & 0.06 & 0.10 & 0.15 \\
\hline
\end{tabular}

The second analysis considers the influence of each personality trait with respect to representations and metrics. The null hypothesis is "the means of the results achieved considering the two values of each trait for the same metrics and representation are equal". The alternative hypothesis consists in asserting that the means are different. The suitable type of t-test is the two-sided one with independent samples because the two groups of testers who generate the results are always different. Also in this case, the significance level is set to 0.1 , for the same reason as before. Table 4 contains the $P$ values computed for this second analysis; most of the values are lower than or equal to the significance level (bold values in the table), except for very few cases; therefore, the null hypothesis can be rejected and personality trait influenced the test results.

3.5.3. Interpretation and Possible Exploitations of the Results. There may be at least two keys of interpretation of the test results shown in Table 2.

Regarding the first interpretation key, the influence of the two values of each personality trait on the results of the design activities can be compared for the same representation and metrics. This key corresponds to a sort of "vertical reading" of Table 2 . For example, agreeable and disagreeable people seem to perform differently from the quantity point of view; the former seem more productive with VR and AR than the latter (3.5 and 4.5 against 2.8 and 3.4, respectively); on the contrary, regarding $\mathrm{PR}$, agreeable people seem less productive than disagreeable ones (3.2 vs. 5.3). In this first key, researchers and designers with limited possibilities in exploiting representations (e.g., $\mathrm{AR}$ and $\mathrm{PR}$ could be unavailable due to the high costs involved; therefore, just VR would be available) could get suggestions on which people to involve, depending on their personalities, to maximize the design efforts or to lead them towards specific characteristics of the expected results (e.g., novel rather than useful, many rather than varying, etc.). Researchers could then investigate the possible relationships between the different traits with respect to the metrics. Designers could exploit these relationships to generate a good team of work involving people already present in the company.

Regarding the second interpretation key, the influence of representations on the results of the design activities can be compared for the same value of personality trait and metrics. This key corresponds to a sort of "horizontal reading" of Table 2. For example, unconscientious people seem able to generate more various $\mathrm{F} / \mathrm{B}$ pairs when dealing with $\mathrm{AR}$ (9.65) than with VR and PR (8.81 and 8.22, respectively) while open to experience people seem able to generate more useful F/B pairs when dealing with AR (0.057) rather than with VR or PR (0.029 and 0.039, respectively). Researchers and designers could exploit this key to investigate about the best representations to use (in terms of products and environments because these are the only elements that discriminate among the representations now) due to specific personalities involved in the project and due to the expected characteristics of the design results. Researchers could start from these suggestions to study how the three remaining elements (interactions 
between environments and products, users and interactions between products and users) could improve even more the design results. Designers could study which representation is the best to select in order to maximize their performance when their goals involve more than one metrics.

It is worth to say that the evaluation of the differences inside each metrics requires particular attention. Recalling that ranges are $[0,+\infty]$ for the quantity, $[1,10]$ for the variety, and $[0,1]$ for novelty and usefulness, it is clear that a difference of some hundredths of the quantity found by two testers or of the variety of two F/B pairs makes no difference but it would mean a lot when dealing with novelty and usefulness. For example, agreeable people performed more or less the same regarding the quantity of $\mathrm{F} / \mathrm{B}$ pairs in VR and $\mathrm{PR}$ (3.5 and 3.2, respectively) while unneurotic people performed much better dealing with VR (0.073) than with AR or PR ( 0.039 and 0.048 , respectively) from the usefulness point of view. In the first case, one-tenth is nothing; in the second case, three-hundredths make a big difference. The orders of magnitude of these values are the same as those used in Sarkar and Chakrabarti's research.

\section{Conclusions}

This research aimed at exploring the influence of personality on shape-based design activities involving different representations. To achieve this, testers showing different personalities performed three tests exploiting three representations. The quantitative and qualitative analyses of the test results confirmed this influence. The research results may improve previous researches already present in literature by highlighting the peculiarities related to personality. For example, Steel et al. [3] claim that conscientious people tend to have more novel ideas; the results here support this but in the specific case of the shape-based design activities, PR limits novelty because of the physicality of the shapes. Considering the work of Kohn and Smith [5] where agreeable people generate fewer ideas, with lower variety, than disagreeable people do, this research confirms it only partially. In fact, agreeable people find fewer solutions only in case of PR; for what concerns VR and AR, they perform better. From the variety point of view, agreeable people find actually solutions showing lower variety than disagreeable people do but this lasts except for PR, where variety is higher. Along with all of this, this research offers different ways to read and exploit the results from both the academic and industrial points of view.

Some research perspectives could be summarized as follows. Personality classification needs to be more objective and precise to increase the granularity and reliability of the results. All of this can consist in considering intermediate values between the current ones for each trait. All representations should be considered rather than only three of them; this would make the research results more robust and their coverage wider. Recent tools like the Mixed Reality Viewer by Microsoft [28] could be used to generate representations to start evaluating if different tools could have influence as well. More people must undergo the tests in order to have more data available to get higher power (probability of rejecting the null hypothesis when the alternative hypothesis is true) from the statistical point of view; moreover, more data would make an in-depth statistical evaluation (considering also representations), as well as the interpretation of the statistical results, feasible and meaningful. Moreover, testers' skill and knowledge could heavily affect the results; thus, some research about this topic is needed as well. It is mandatory to investigate how combinations of personality traits influence design instead of considering them as independent from each other as it is now. Different design activities than the shapebased must be considered to check if the coverage of the results obtained here can be made wider or, on the contrary, if each design activity has its own peculiarities from this point of view. Finally, strictly speaking, current computation of variety does not fully support statistical analyses because the variety values are dependent from each other. In fact, variety values are assigned to every $\mathrm{F} / \mathrm{B}$ pair depending on how many times the same F/B pair is found by other testers sharing same values of personality traits. This aspect pushes towards searching new ways to calculate variety in order to make statistical analyses feasible. In addition, other metrics than the five considered here should be involved, traditional as well as more recent, like the satisfaction and involvement of the testers in the interaction with the products, etc.

\section{Data Availability}

The data used to support the findings of this study are included within the article.

\section{Conflicts of Interest}

The authors declare that there are no conflicts of interest regarding the publication of this paper.

\section{Acknowledgments}

The authors would like to thank Eng. Marco Sortino and Eng. Emanuele Vaglio for their help in generating the material to perform the PR test.

\section{References}

[1] S. Y. Sung and J. N. Choi, "Do big five personality factors affect individual creativity? The moderating role of extrinsic motivation," Social Behavior and Personality, vol. 37, no. 7, pp. 941-956, 2009.

[2] S. Filippi and D. Barattin, "Evaluating the influences of heterogeneous combinations of internal/external factors on product design," in Proceedings of the 21st International Conference on Engineering Design ICED '17, vol. 8, pp. 1-10, Vancouver; Canada, August 2017, no. DS87-8.

[3] G. D. Steel, T. Rinne, and J. Fairweather, "Personality, nations, and innovation: relationships between personality traits and national innovation scores," Cross-Cultural Research, vol. 46, no. 1, pp. 3-30, 2012.

[4] S. Rothmann and E. P. Coetzer, "The big five personality dimensions and job performance," Journal of Industrial Psychology, vol. 29, no. 1, pp. 68-74, 2003. 
[5] N. W. Kohn and S. M. Smith, "Collaborative fixation: effects of others' ideas on brainstorming," Applied Cognitive Psychology, vol. 25, pp. 359-371, 2011.

[6] S. Filippi and D. Barattin, "Influence of representations on shape-based design activities," International Journal on Interactive Design and Manufacturing (IJIDeM), vol. 13, no. 1, pp. 277285, 2019.

[7] E. M. Starkey, A. S. McKay, S. T. Hunter, and S. R. Miller, "Dissecting creativity: how dissection virtuality, analogical distance, and product complexity impact creativity and selfefficacy," in Proceedings of the 7th International Conference on Design Computing and Cognition DCC '16, pp. 63-82, Chicago, USA, June 2016.

[8] M. L. Maher, L. Lee, J. Gero, R. Yu, and T. Clausner, "Characterizing tangible interaction during a creative combination task," in Proceedings of the 7th International Conference on Design Computing and Cognition DCC '16, pp. 43-62, Chicago, USA, June 2016.

[9] R. J. Youmans, "The effects of physical prototyping and group work on the reduction of design fixation," Design Studies, vol. 32, no. 2, pp. 115-138, 2011.

[10] T. Kohler, K. Matzler, and J. Füller, "Avatar-based innovation: using virtual worlds for real-world innovation," Technovation, vol. 29, no. 6-7, pp. 395-407, 2009.

[11] S. Filippi and D. Barattin, Involving Autism Spectrum Disorder (ASD) Affected People in Design, Lecture Notes in Mechanical Engineering, 2017.

[12] S. Karimi and M. R. Kangavari, "A Computational Model of Personality," Procedia - Social and Behavioral Sciences, vol. 32, pp. 184-196, 2012.

[13] R. B. Cattell, "The description of personality: basic traits resolved into clusters," Journal of Abnormal and Social Psychology, vol. 38, no. 4, pp. 476-506, 1943.

[14] E. C. Tupes and R. E. Christal, "Recurrent personality factors based on trait ratings," Tech. Rep. ASD-TR-61-97, Lackland Air Force Base, TX. U.S. Air Force, 1961.

[15] J. M. Digman and J. Inouye, "Further Specification of the Five Robust Factors of Personality," Journal of Personality and Social Psychology, vol. 50, no. 1, pp. 116-123, 1986.

[16] R. R. McCrae and P. T. Costa, "Validation of the five-factor model of personality across instruments and observers," Journal of Personality and Social Psychology, vol. 52, no. 1, pp. 81-90, 1987.

[17] L. R. Goldberg, "An alternative 'description of personality': the big-five factor structure," Journal of Personality and Social Psychology, vol. 59, no. 6, pp. 1216-1229, 1990.

[18] J. Mikkonen and Y. T. Hsieh, "Prototyping with experience workshop," in Proceedings of the International Conference on Human-Computer Interaction, vol. 8012, pp. 564-572, Las Vegas, USA, July 2013.

[19] Alessi, “The Italian factory of industrial design," 2018, https:// www.alessi.com/us_en/.

[20] C. Rogers, J. Lau, D. Huynh et al., "Capturing the perceived phantom limb through virtual reality," Advances in Human Computer Interaction, vol. 2016, Article ID 8608972, 6 pages, 2016.

[21] G. Alce, M. Wallergard, and K. Hermodsson, "WozARd: a wizard of Oz method for wearable augmented reality interaction-a pilot study," Advances in Human Computer Interaction, vol. 2015, Article ID 271231, 10 pages, 2015.

[22] F. P. W. Melchels, J. Feijen, and D. W. Grijpma, "A review on stereolithography and its applications in biomedical engineering," Biomaterials, vol. 31, no. 24, pp. 6121-6130, 2010.
[23] P. Sarkar and A. Chakrabarti, "Assessing design creativity," Design Studies, vol. 32, no. 4, pp. 348-383, 2011.

[24] "HoloLens by Microsoft," 2018, https://www.microsoft.com/ en-us/hololens.

[25] Ultimaker 2+ by Ultimaker, 2018, https://ultimaker.com/en/ products/ultimaker-2-plus.

[26] C. J. Soto and O. P. John, "Ten facet scales for the Big Five Inventory: convergence with NEO PI-R facets, self-peer agreement, and discriminant validity," Journal of Research in Personality, vol. 43, no. 1, pp. 84-90, 2009.

[27] D. C. Montgomery and G. C. Runger, Applied Statistics and Probability for Engineers, John Wiley and Sons, Inc, New Jersey, NJ, USA, 3rd edition, 2002.

[28] Mixed Reality Viewer by Microsoft, 2018, https://www.microsoft .com/it-it/p/3d-viewer/9nblggh42ths?activetab=pivot:overviewtab. 


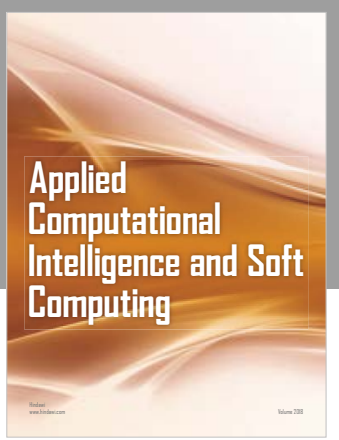

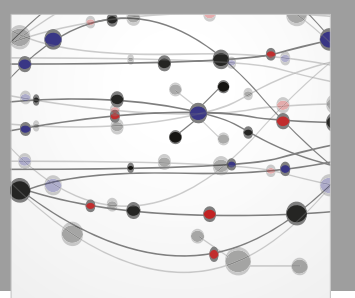

The Scientific World Journal
Submit your manuscripts at

Computing
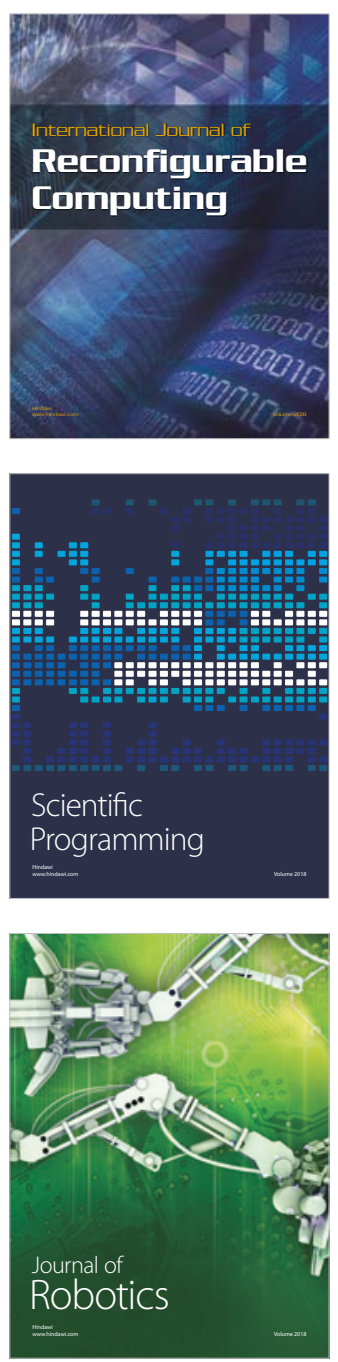

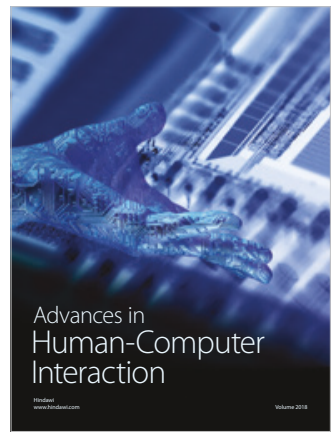

Human-Compute

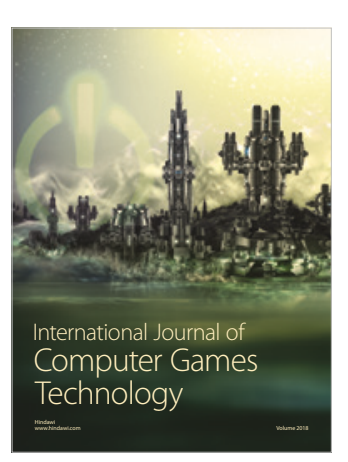

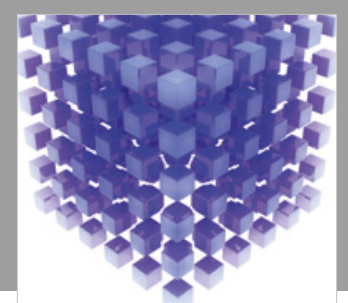

Mathematical Problems in Engineering

\section{Engincering}
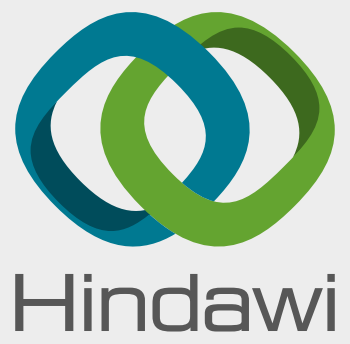

www.hindawi.com
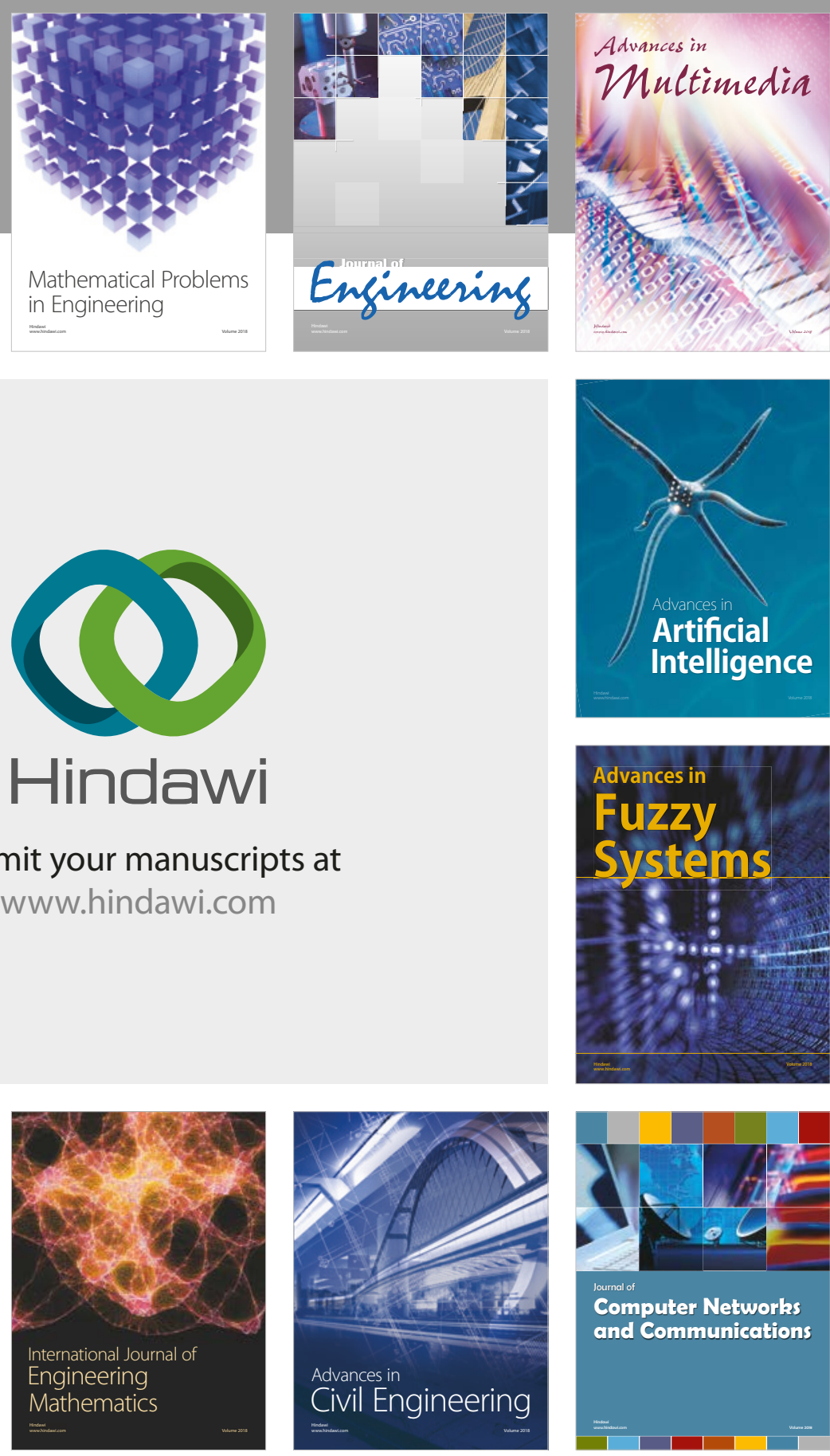

Computer Networks and Communications

Multimedia
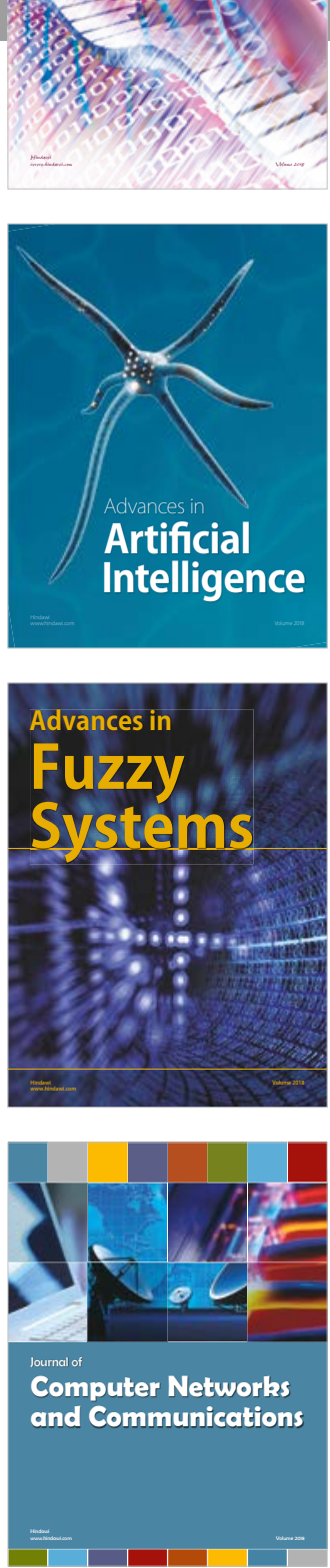

Advances in

Modelling \&

Simulation

in Engineering

interaction

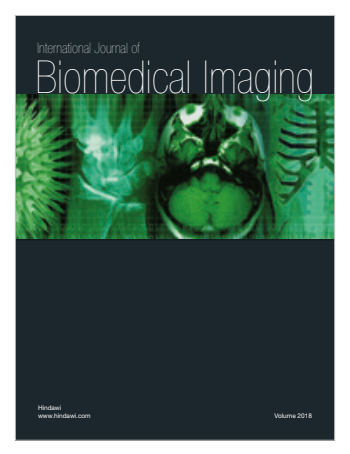

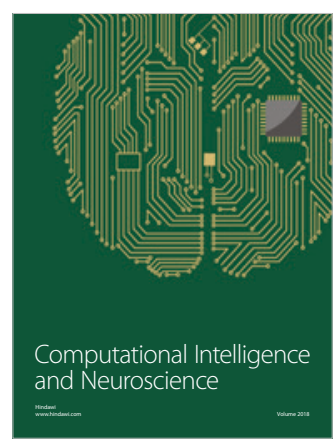

